\title{
Communication
}

\section{High-Sensitivity Phase Detection of Reflection-Type Guided-Mode Resonance Sensor Based on Rotating Azimuth Angle Using a Heterodyne Interferometer}

\author{
Jaturon Tongpakpanang and Wen-Kai Kuo *
}

check for updates

Citation: Tongpakpanang, J.; Kuo, W.-K. High-Sensitivity Phase Detection of Reflection-Type Guided-Mode Resonance Sensor Based on Rotating Azimuth Angle Using a Heterodyne Interferometer. Photonics 2022, 9, 91. https:// doi.org/10.3390/photonics9020091

Received: 7 January 2022

Accepted: 31 January 2022

Published: 3 February 2022

Publisher's Note: MDPI stays neutral with regard to jurisdictional claims in published maps and institutional affiliations.

Copyright: (c) 2022 by the authors. Licensee MDPI, Basel, Switzerland. This article is an open access article distributed under the terms and conditions of the Creative Commons Attribution (CC BY) license (https:// creativecommons.org/licenses/by/ $4.0 /)$.
Graduate Institute of Electro-Optical and Materials Science, National Formosa University, Yunlin 63208, Taiwan; d0677104@gm.nfu.edu.tw

* Correspondence: wkkuo@nfu.edu.tw

\begin{abstract}
A high-sensitivity phase-detection system is proposed for a reflection-type guided-mode resonance (GMR) sensor, which achieves the resonance condition by rotating the azimuth angle and utilizes an electro-optic (EO) heterodyne interferometer. By rotating the GMR sensor azimuthally, the direction of the reflected light can be maintained in reflection-type detection, and the optical system can be compactly constructed because the light-tracking rotation stage is not required. The phase-detection sensitivity can be enhanced in this common-path EO heterodyne interferometer by rotating the analyzer in front of the photodetector; therefore, this system can achieve both a high sensitivity and low limit of detection. Numerical and experimental results of the reflectivity and phase response curves versus the azimuth angle were compared. The proposed system was used to perform gas sensing, and its detection sensitivity and limit were $3.73 \times 10^{4} \mathrm{deg} / \mathrm{RIU}$ and $2.68 \times 10^{-7}$ RIU, respectively.
\end{abstract}

Keywords: guided-mode resonance; conical diffraction; wave plates; refractive index sensing

\section{Introduction}

The guided-mode resonance (GMR) effect in a periodic dielectric waveguide has been widely studied and applied to various optical devices. GMR devices utilize a periodic grating to couple incident light to the waveguide structure. When the diffraction wave is phase-matched to a leaky mode, GMR devices can produce total reflection or transmission peaks [1]. Because this matching condition is highly sensitive to the refractive index (RI) of material on the GMR grating's surface, GMR devices based on resonance wavelength shift detection have been used as biosensors [2,3]. Additionally, the phase of GMR devices has been reported to change abruptly near the resonance condition [4]. An optical retarder based on GMR has been proposed, and a phase shift of $\pi$ at the operating wavelength was achieved [5]. Using the dramatic phase-change near resonance, many phase-based interferometric sensing approaches have been demonstrated. In our previous study, a transmitted-type GMR sensor based on phase detection was proposed using an electrooptic (EO) heterodyne interferometer [6]. The phase curves of two orthogonally polarized components ( $p$ - and s-polarized) were plotted against various incident angles. Subsequently, the same sensor, but with the use of a phase-shift interferometer, was reported [7]. In both approaches, the GMR sensor is rotated to a resonance incident angle to optimize the phase-detection sensitivity and the analyzer can be tuned to further enhance detection sensitivity. In this transmitted-type configuration, the incident light passes through the GMR sensor. When the GMR sensor is rotated, the direction of the transmitted light is maintained and the position of the light-receiving device can be fixed at the same position. This results in a simple and compact optical phase-detection system. Conversely, in a reflection-type configuration, when the GMR device is rotated to be a resonant incident, the direction of the reflected light changes, and the position of the light-receiving device must 
be changed accordingly. This complicates the phase-detection system because it requires a $\Delta-2 \theta$ motorized rotation stage for tracking the reflected light. Additionally, in some sensing applications, the reflection-type configuration is required to prevent the incident light from passing through the sample on the GMR sensor, as this may result in unexpected effects in the tested samples.

Recently, a reflection-type common-path interferometer based on a Wollaston prism was developed, and a GMR device was designed with various grating pitches to ensure that the resonance occurred at normal incidence [8]. Due to the common-path interferometer, this system was not sensitive to environmental noise and achieved a low detection limit. Because the resonance condition of a GMR device can also be achieved by changing the azimuth angle, GMR filters under conical mounting have been studied $[9,10]$. Based on this concept, a GMR sensor with reflection-type phase detection using a Mach-Zehnder interferometer was proposed to maintain the direction of the reflected light and achieve improved resonance by altering the azimuth angle of the GMR device [11]. In this approach, resonance was attained by rotating the azimuth angle to be a band-pass filter with a center wavelength similar to a He-Ne laser, and the phase-detection sensitivity was compared under resonance and non-resonance conditions. However, the phase properties of scanned azimuth angles for different incident angles were not investigated in their study. In this paper, details of the reflection-type GMR sensor based on azimuthal rotation are reported. The azimuth angle was scanned at various incident angles to construct phase curves, which were numerically studied, and the experimental results obtained using an EO heterodyne interferometer are presented. Furthermore, the phase-detection sensitivity was enhanced by rotating the analyzer in the EO heterodyne interferometer, and the measurement results of a GMR gas sensor are reported.

\section{Simulation of the Guided-Mode Resonance Device}

A schematic of the GMR device structure under conical mounting is shown in Figure 1. Its structure comprises a one-dimensional (1D) sinusoidal UV glue grating imprinted on a glass substrate and a waveguide layer formed of over-coated, aluminum-doped zinc oxide (AZO) thin film. Its fabrication was explored in a previous study [6]. The grating pitch is defined by the period $\Lambda$ along the $x$-axis. Polarized light is obliquely incident on the back-side substrate of the GMR device. For conical mounting, the incident wave vector $\mathrm{k}$ is a function of both the angle of incidence $\theta$ and the azimuth angle $\varphi$. The conically diffracted wave vector $\mathrm{km}$ can be represented by the $m$ th-order diffraction component given as in [9]:

$$
k_{m}=k_{x, m} \hat{x}+k_{y, m} \hat{y}
$$

where $k_{x, m}$, and $k_{y, m}$ are the components along the $\mathrm{x}$ - and $\mathrm{y}$-axes, respectively. For 1D-GMR, $k_{x, m}$, and $k_{y, m}$ can be expressed as:

$$
\begin{gathered}
k_{x, m}=-k \sin \dot{\theta} \cos \varphi+m\left(\frac{2 \pi}{\Lambda}\right) \\
k_{y, m}=-k \sin \dot{\theta} \cos \varphi
\end{gathered}
$$

Resonance can occur when the propagation constant in the leaky waveguide is equal to the longitudinal component of the $m$-th order diffracted wave vector $\mathrm{km}$. Thus, the phase matching of the diffracted wave and the leaky mode of the waveguide can be achieved by rotating the azimuth angle. The properties of GMR in response to its incident and azimuth angles were verified utilizing commercial software (Rsoft, DiffractMOD) based on the rigorous coupled-wave analysis (RCWA) method.

In the simulations, the refractive indices (RIs) of the glass substrate, UV glue grating, and AZO film were $n_{s}=1.515, n_{g}=1.475$, and $n_{A Z O}=1.79$, respectively. The grating period $\Lambda$ was $555 \mathrm{~nm}$, corresponding to a commercially available holographic grating with 1800 lines/mm, and the modulation depth of the UV glue grating was $75 \mathrm{~nm}$, based on 
an atomic force microscope (AFM) measurement result. An AZO film with a thickness of $275 \mathrm{~nm}$ was used as a high-RI waveguide and air $(n=1.0)$ was used as the superstrate. A He-Ne laser with a wavelength of $632.8 \mathrm{~nm}$ was incident on the substrate at an angle of $\theta$, and the effect of the change in incident angle on the zero-order reflection was first analyzed for both $p$ - and s-polarization. The reflectance maps of the GMR device are shown in Figure 2. The dark blue area represents regions with low reflectivity, whereas the red area represents regions with high reflectivity. The azimuth angle varies from $-45^{\circ}$ to $45^{\circ}$ for selected incident angles ranging from $28^{\circ}$ to $33^{\circ}$. Figure $2 \mathrm{a}, \mathrm{b}$ illustrate the reflectivity maps of the GMR device for $p$ - and s-polarized light, respectively. When the incident angle was in the range $28^{\circ}-31^{\circ}$, only $p$-polarization exhibited resonance, whereas s-polarization could be used as a reference in the heterodyne interferometer. In this experiment, the incident angle was set between $28^{\circ}$ and $31^{\circ}$, and then the azimuth angle was rotated to obtain the phase difference between $p$ - and s-polarization. In addition, the reflectance pattern in Figure $2 \mathrm{a}$ can also be found in Figure $2 \mathrm{~b}$ with a weak reflectance and vice versa. This reveals a coupling between the TE and TM modes of the grating waveguide.

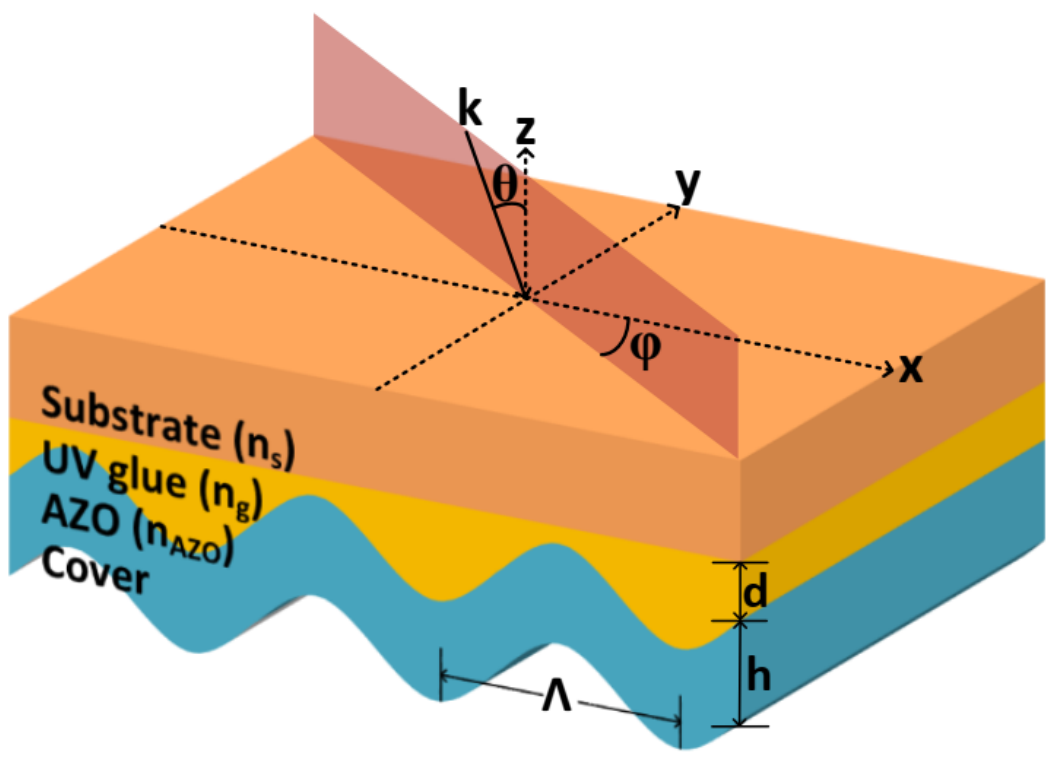

Figure 1. Schematic of the guided-mode resonance device structure under conical mounting.

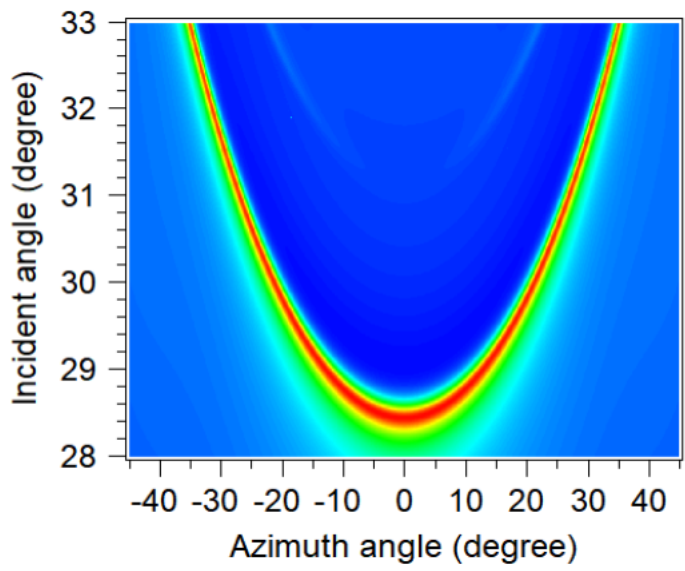

(a)
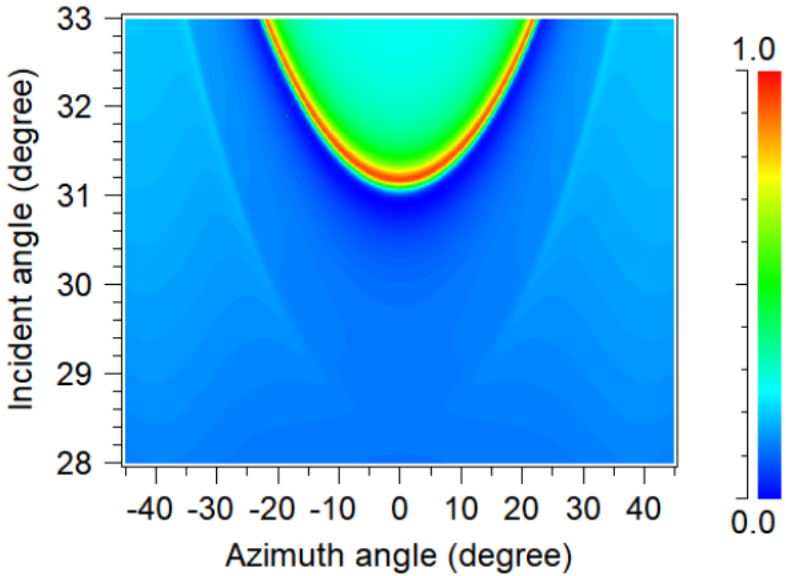

(b)

Figure 2. Angular-resolved reflectivity maps of GMR under conical mounting for (a) $p$ - and (b) s-polarization. 
Plots of the reflectivity versus the azimuth angle for three different incident angles, $29.5^{\circ}, 30.0^{\circ}$, and $30.5^{\circ}$, are shown in Figure $3 \mathrm{a}$. As the incident angle increased, the resonant azimuth angle also increased, and the resonant angle width decreased, as shown in Figure 2. Weak resonances were observed for s-polarization. The phase response of the reflected light versus the azimuth angle for different incident angles is shown in Figure $3 \mathrm{~b}$. The $p$-polarization phase curves changed dramatically, corresponding to the resonance peak shown in Figure 2a. On the other hand, the s-polarization phase curves exhibited only small changes and could be used as a reference in the heterodyne interferometer. Plots of the phase differences between the $s$ - and $p$-polarization components are shown in Figure 3c.

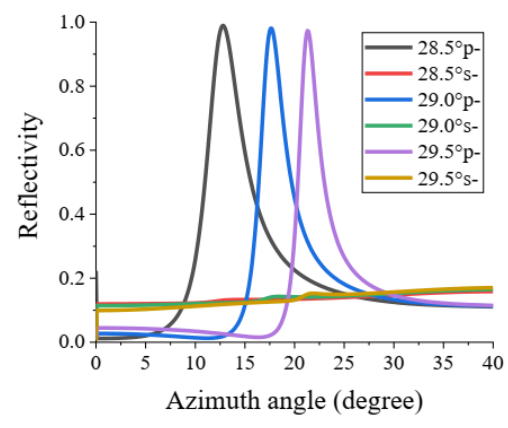

(a)

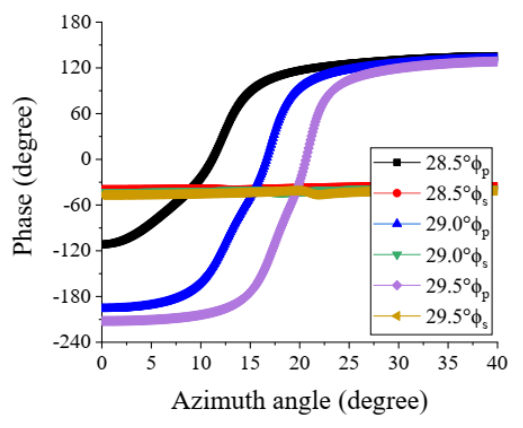

(b)

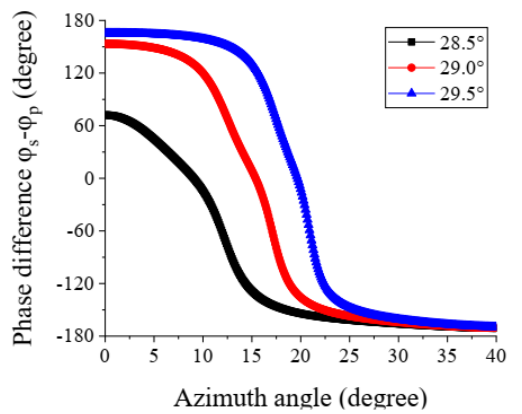

(c)

Figure 3. (a) Reflectivity and (b) phase curves for the $p$ - and s-polarized components of the scanned azimuth angle, and (c) plots of phase differences $\left(\varphi_{s}-\varphi_{p}\right)$ between $s$ - and $p$-polarization at three different incident angles $\left(28.5^{\circ}, 29.0^{\circ}\right.$, and $\left.29.5^{\circ}\right)$.

\section{Experimental Results}

The GMR device was fabricated using the nanoimprinting process, and details of this process can be found in the previous study [6]. Instead of coating titanium oxide (TiO2), an AZO film was used in this study because of the convenience of the coating machine. The AFM surface morphology of the fabricated GMR device is shown in Figure 4a. The GMR device, equipped with a gas chamber and mounted on a rotation stage to scan the azimuth angle, is shown in Figure $4 \mathrm{~b}$. Inlet and outlet tubes were used to control the gas flow inside the chamber. A schematic of the reflection-type, phase difference detection system based on the EO heterodyne interferometer is shown in Figure 4c. Details of the EO heterodyne interferometer also can be found in the previous study [6]. In this setup, a lock-in amplifier was used to record the interference signal intensity, as well as the phase difference between the interference signal and reference signal from the signal generator to drive the $\mathrm{EO}$ modulator $(\mathrm{EOM})$ with a frequency of $100 \mathrm{~Hz}$. The phase reading value was equal to the phase difference between the $s$ - and $p$-polarization components.

In the first experiment, the EOM and the analyzer were removed from the interferometer, and an optical power detector (Thorlabs PM100) was used in place of the photodetector. The reflectance curves of the scanning azimuth angles for different incident angles were obtained to validate the simulation results. The measured reflectivity of the GMR sensor for $p$ - and s-polarization is shown in Figure 5a. The results reveal that only $p$-polarized lights produce peaks at the resonance azimuth angles, and when the incident angle increases, the resonance azimuth angle also increases. This is consistent with the simulation results shown in Figure 3a. However, it seems that the resonance angles, widths, and three peak values of the measured results are not the same as the simulation results. These may be due to the nonuniform grating structure, and the probing light position on the device may change during scanning the azimuth angle, whereas these results would not affect the phase measurement. Subsequently, all the removed components were installed as the set-up shown in Figure 4c, and this system was used to measure the phase difference responses of scanned azimuth angles with three different incident angles. The results are shown in 
Figure 5b, which roughly agree with the simulation results, as shown in Figure 3c. The increases in the ends of the phase curves may also be due to the light probing position change during the scanning of the azimuth angle.

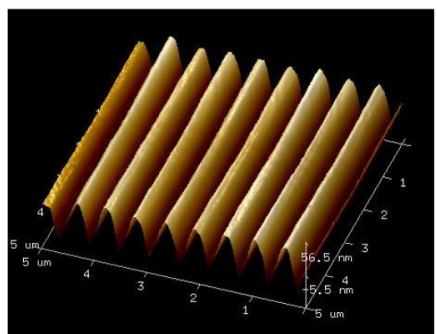

(a)

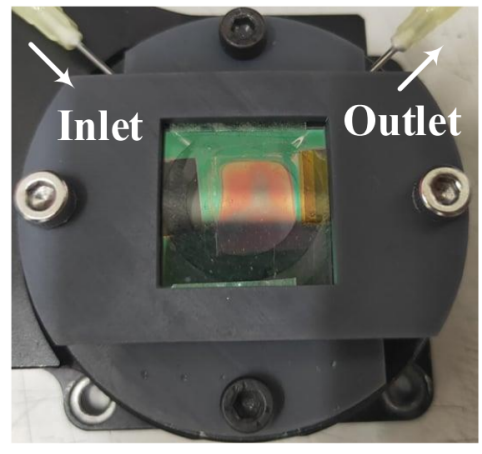

(b)

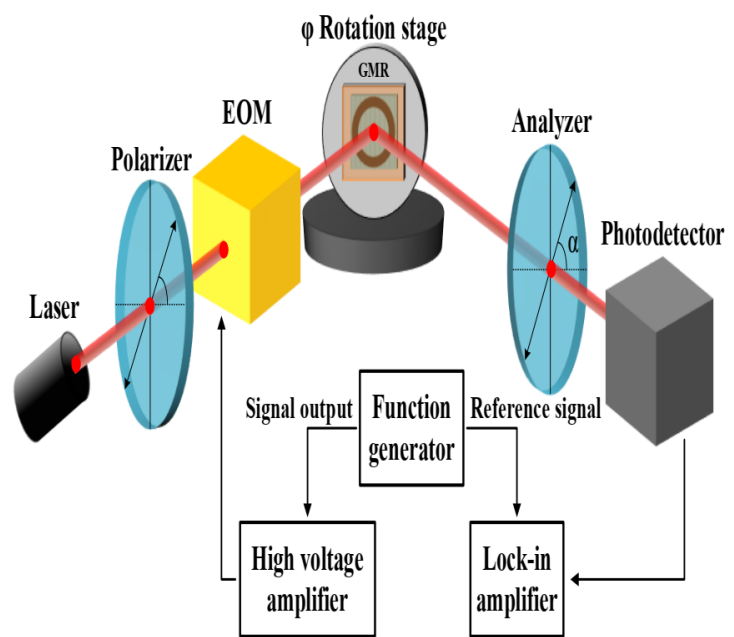

(c)

Figure 4. (a) AFM surface morphology of the fabricated GMR device. (b) Photograph of the GMR gas sensor mounted on a rotation stage. (c) Schematic of the reflection-type, phase difference detection system based on an electro-optic heterodyne interferometer.

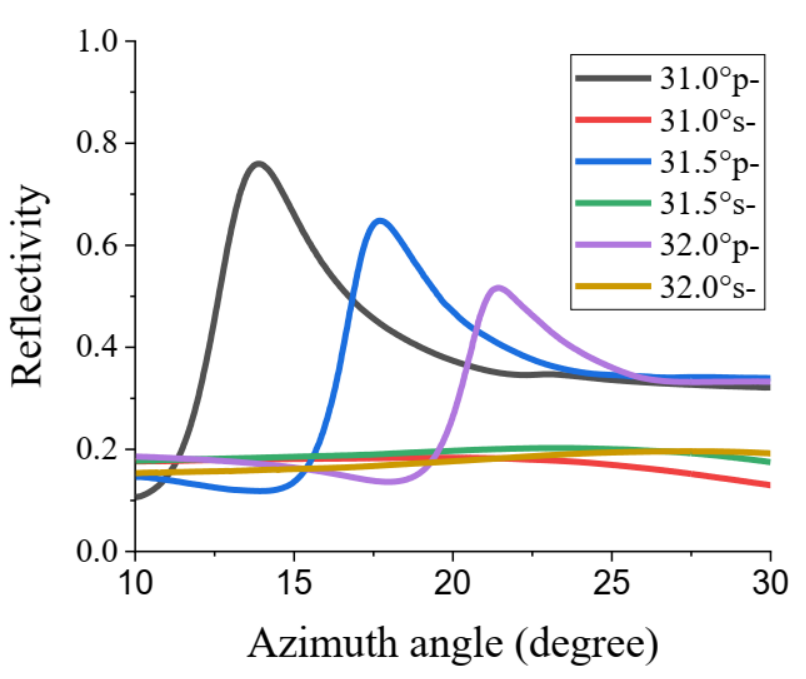

(a)

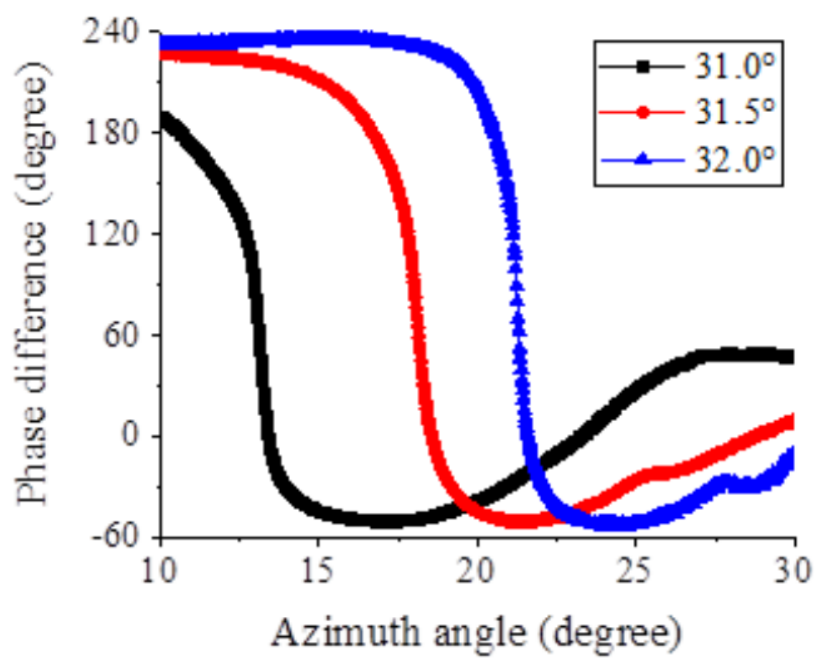

(b)

Figure 5. Measured (a) reflectivity and (b) phase difference of the reflection-type GMR sensor for sand $p$-polarized light versus the azimuth angle with three different incident angles.

In the second experiment, the GMR device was used as a gas sensor. Before performing the sensing measurement, tunable reflected phase-detection sensitivity was demonstrated. In the EO heterodyne interferometer, the phase-detection sensitivity can be tuned by 
rotating the analyzer. In this experiment, the incident angle was set at $31.7^{\circ}$, and the measurement results of the lock-in amplitude and phase curves versus the azimuth angle for three analyzer angles $\left(45^{\circ}, 47^{\circ}\right.$, and $\left.49^{\circ}\right)$ are shown in Figure $6 a, b$, respectively. This reveals that, near the resonance angle, the phase slope increases when the lock-in amplitude decreases. Rotating the analyzer can tune the passing-through light amplitude ratio between $s$ - and $p$-polarization components, and hence change the interference of light modulation intensity on the photodetector. A small lock-in amplitude reading means less modulation of the light intensity. The decrease in modulation can enhance phase change; however, the modulation strength cannot be tuned too small and must satisfy an acceptable signal-to-noise ratio (SNR). Consequently, the analyzer was set to $49^{\circ}$ to obtain an acceptable SNR; this obtained the steepest slope on the phase curve, and hence the highest sensitivity. According to the results in Figure $6 \mathrm{~b}$, the azimuth angle was rotated to $20.45^{\circ}$ in the $\mathrm{EO}$ heterodyne interferometer.

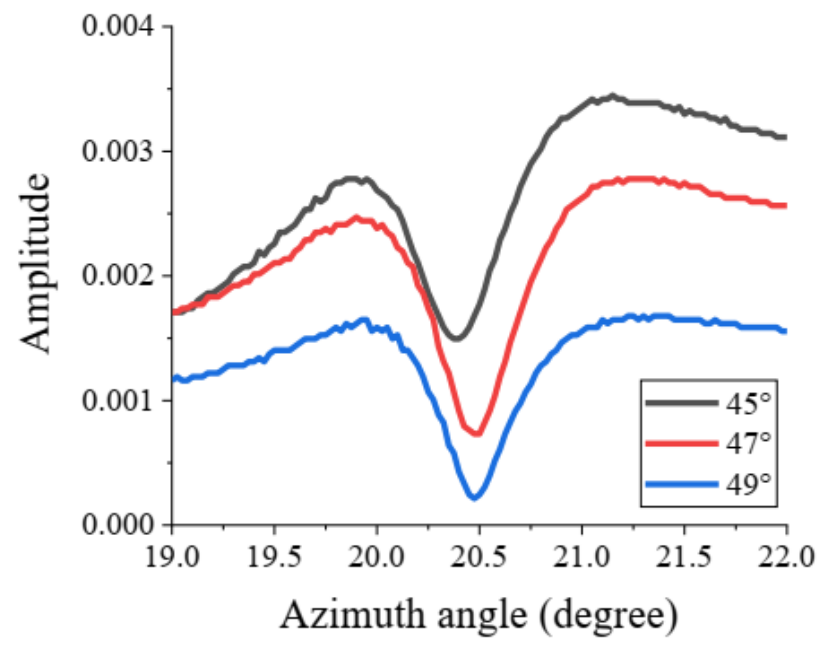

(a)

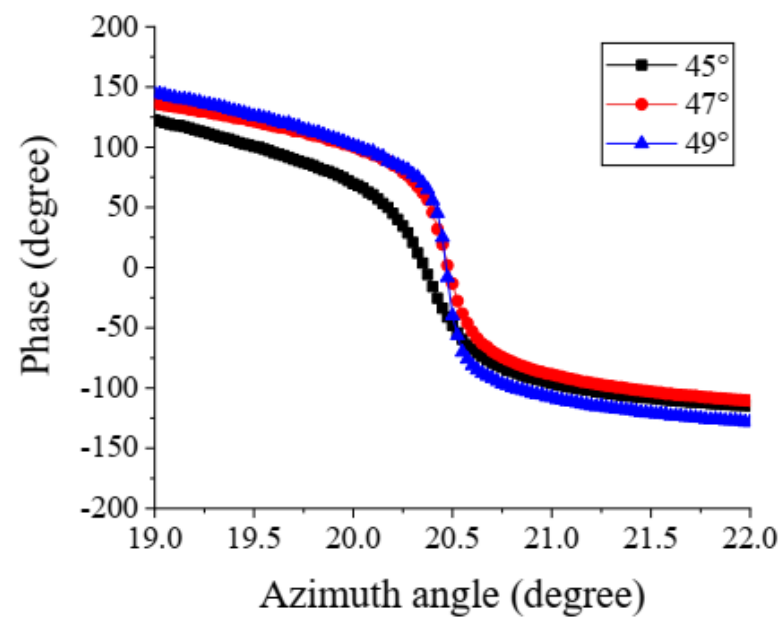

(b)

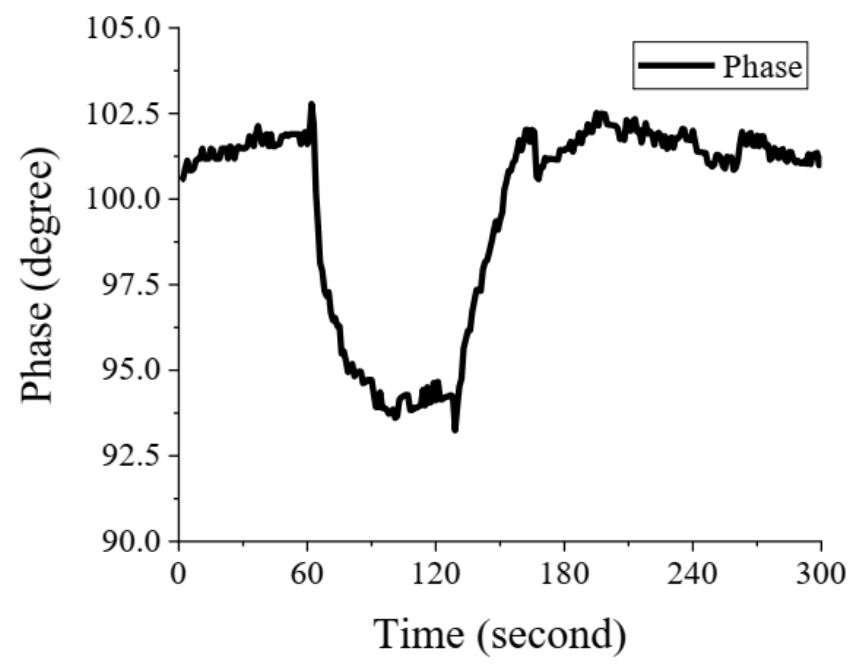

(c)

Figure 6. Measured lock-in (a) amplitude and (b) phase curves versus the azimuth angle for three different analyzer rotation angles, $45^{\circ}, 47^{\circ}$, and $49^{\circ}$. (c) Time evolution of the phase value with injecting He gas from $\mathrm{t}=60$ to $120 \mathrm{~s}$ at an analyzer angle of $49^{\circ}$. 
Subsequently, a helium (He) gas was injected into the chamber to replace the air on the GMR sensor surface. The RIs of air and helium are approximately 1.000276 (for dry air at $15{ }^{\circ} \mathrm{C}$ and 101,325 Pa with $450 \mathrm{ppm} \mathrm{CO}$ content) and 1.000035, respectively, at a wavelength of $680 \mathrm{~nm}$ [12]. The time evolution results of the phase curve are shown in Figure 6c. In this experiment, He gas was continuously injected from $t=60$ to $120 \mathrm{~s}$. The measured phase decreased at the moment of injection because of the decrease in RI on the GMR sensor surface. The phase then increased as the He gas leaked out. Because this system did not maintain the same temperature in the chamber during the measurement, some small variations in the resulting curve were observed. The phase change during the He injection was approximately $9^{\circ}$, and the RI change $\Delta n=2.41 \times 10^{-4}$ RIU. Consequently, in this measurement, the proposed system achieved a detection sensitivity of $3.73 \times 10^{4} \mathrm{deg}$. $/ \mathrm{RIU}$. The fluctuation of phase may be caused by thermal and environment noise. However, this value may be further improved by increasing the time constant of the lock-in amplifier. This may require more time to complete the measurement. Assuming that the statistical error phase of the lock-in amplifier was $0.01^{\circ}$, its detection limit was $2.68 \times 10^{-7}$ RIU.

\section{Conclusions}

In this study, the phase properties of scanned azimuth angles for different incident angles were numerically investigated for the first time. Subsequently, a high-sensitivity phase-detection system for a reflection-type GMR sensor was successfully demonstrated. The incident angle could be approximately set within an angle range, then the azimuth angle was rotated to obtain resonance without altering the reflective light direction. Furthermore, the analyzer was tuned to achieve a higher detection sensitivity. These results show that a highly sensitive yet compact phase-detection system for reflection-type GMR sensors can be achieved, which may have great potential in biosensing instruments.

Author Contributions: Conceptualization, W.-K.K.; methodology, J.T.; writing-original draft preparation, J.T.; writing-review and editing, W.-K.K.; visualization, J.T.; supervision, W.-K.K.; project administration, W.-K.K.; funding acquisition, W.-K.K. All authors have read and agreed to the published version of the manuscript.

Funding: This research was funded by the Ministry of Science and Technology, Taiwan, grant number 109-2221-E-150-07, and 110-2221-E-150-027.

Institutional Review Board Statement: Not applicable.

Informed Consent Statement: Not applicable.

Data Availability Statement: Not applicable.

Acknowledgments: The authors thank the Ministry of Science and Technology, Taiwan for the funding support.

Conflicts of Interest: The authors declare no conflict of interest.

\section{References}

1. Ding, Y.; Magnusson, R. Resonant leaky-mode spectral-band engineering and device applications. Opt. Express 2004, 12, 5661-5674. [CrossRef] [PubMed]

2. Cunningham, B.; Lin, B.; Qiu, J.; Li, P.; Pepper, J.; Hugh, B. A plastic colorimetric resonant optical biosensor for multiparallel detection of label-free biochemical interactions. Sens. Actuat. B Chem. 2002, 85, 219-226. [CrossRef]

3. Ferrie, A.M.; Wu, Q.; Fang, Y. Resonant waveguide grating imager for live cell sensing. Appl. Phys. Lett. 2010, 97, 357. [CrossRef] [PubMed]

4. Magnusson, R.; Shokooh-Saremi, M.; Wang, X. Dispersion engineering with leaky-mode resonant photonic lattices. Opt. Exp. 2010, 18, 108-116. [CrossRef] [PubMed]

5. Magnusson, R.; Shokooh-Saremi, M.; Johnson, E.G. Guided-mode resonant wave plates. Opt. Lett. 2010, 35, 2472-2474. [CrossRef] [PubMed]

6. Kuo, W.K.; Huang, N.C.; Weng, H.P.; Yu, H.H. Tunable phase detection sensitivity of transmitted-type guided-mode resonance sensor in a heterodyne interferometer. Opt. Express 2014, 22, 22968-22973. [CrossRef] [PubMed] 
7. Kuo, W.K.; Syu, S.H.; Lin, P.Z.; Yu, H.H. Tunable sensitivity phase detection of transmitted-type dual-channel guided-mode resonance sensor based on phase-shift interferometry. Appl. Opt. 2016, 55, 903-907. [CrossRef] [PubMed]

8. Barth, I.; Conteduca, D.; Reardon, C.; Johnson, S.; Krauss, T.F. Common-path interferometric label-free protein sensing with resonant dielectric nanostructures. Light Sci. Appl. 2020, 9, 96. [CrossRef] [PubMed]

9. Lacour, D.; Granet, G.; Plumey, J.P.; Mure-Ravaud, A. Polarization independence of a one-dimensional grating in conical mounting. J. Opt. Soc. Am. A 2003, 20, 1546-1552. [CrossRef] [PubMed]

10. Yukina, R.; Sahoo, P.K.; Sharma, J.; Takamura, T.; Joseph, J.; Sandhu, A. Wide wavelength range tunable one-dimensional silicon nitride nanograting guided mode resonance filter based on azimuthal rotation. AIP Adv. 2017, 7, 015313. [CrossRef]

11. Sahoo, P.K.; Sarkar, S.; Joseph, J. High sensitivity guided-mode-resonance optical sensor employing phase detection. Sci. Rep. 2017, 7, 7607. [CrossRef] [PubMed]

12. Weber, M.J. Handbook of Optical Materials; CRC Press: Boca Raton, FL, USA, 2003. 